\title{
Tumor progression locus 2 ablation suppressed hepatocellular carcinoma development by inhibiting hepatic inflammation and steatosis in mice
}

\author{
Xinli Li ${ }^{1,4}$, Chun Liu', Blanche C. Ip ${ }^{1}$, Kang-Quan Hu', Donald E. Smith², Andrew S. Greenberg ${ }^{3}$ \\ and Xiang-Dong Wang ${ }^{1 *}$
}

\begin{abstract}
Background: Tumor progression locus 2 (TPL2), a serine-threonine kinase, functions as a critical regulator of inflammatory pathways and mediates oncogenic events. The potential role of $T p / 2$ in nonalcoholic fatty liver disease (NAFLD) associated hepatocellular carcinoma (HCC) development remains unknown.

Methods: Both wild-type and Tp/2 knockout male mice were initiated by a hepatic carcinogen (diethylnitrosamine, i.p. with a single dose of $25 \mathrm{mg}^{\mathrm{kg}}{ }^{-1}$ )at 2 weeks of age, and then were given the high carbohydrate diet feeding to induce hepatic steatosis, inflammation, adenoma and HCC for 24 weeks.

Results: Tp/2 knockout mice had significantly lower incidences of liver tumor and developed hepatocellular adenoma only, which is contrast to wild-type mice where they all developed HCC. Tp/2 knockout mice had significantly downregulated phosphorylation of JNK and ERK, and levels of mRNA expression of pro-inflammatory cytokines (II-1, $1 /-18$, Mcp-1 and Nalp3), which correlated with the reduced incidence and number of hepatic inflammatory foci. Furthermore, Tpl2 ablation resulted in decreased hepatic steatosis and expression of de novo lipogenesis related markers (ACC, SCD1, SREBP1C and AKT phosphorylation), as well as reduction of endoplasmic reticulum stress biomarkers PERK and elF-2a.
\end{abstract}

Conclusion: The study revealed for the first time that Tp/2 plays a significant role in promoting HCC development by its pro-inflammatory effect, which suggested that Tp/2 could be a molecular target for HCC prevention.

Keywords: TPL2, HCC, Tumorigenesis, Inflammation, Steatosis

\section{Background}

Hepatocellular carcinoma (HCC) is the sixth most prevalent human malignancy in the world and the third leading cause of cancer-related mortality [1]. Relative 5 -year survival rate of $\mathrm{HCC}$ is only $15 \%$, which emphasizes the importance of primary prevention of HCC. Chronic infection by hepatitis $B$ and hepatitis $C$ virus, exposure to aflatoxin, alcoholic injury and genetic disorders have proven to play a critical role in the development of HCC [2], however, the etiology remains

\footnotetext{
* Correspondence: xiang-dong.wang@tufts.edu

${ }^{1}$ Nutrition and Cancer Biology Laboratory, Jean Mayer USDA Human

Nutrition Research Center on Aging at Tufts University, 711 Washington Street, Boston, MA 02111, USA

Full list of author information is available at the end of the article
}

unknown in almost $50 \%$ of HCC patients. Recent studies suggest that nonalcoholic fatty liver disease (NAFLD) is associated with an increased risk of HCC $[3,4]$, but it remains unclear whether NAFLD is a causative factor for HCC [5].

Steatosis is the initial stage of NAFLD, which can progress into more pathological stages including nonalcoholic steatohepatitis (NASH), fibrosis and cirrhosis, with the result of the increased risk for HCC development. Previous studies had indicated the significant contribution of NASH to HCC development, where pro-inflammatory cytokine and chemokines favors malignant transformation of hepatocytes by providing a tumor microenvironment $[6,7]$. Inflammatory cascades through interactions of numerous signaling pathways progressively stimulated hepatocyte 
proliferation and apoptosis [8]. However, the potential causal relationships among inflammation, steatosis and HCC development need more supporting evidence.

Tumor progression locus 2 (TPL2), a serine-threonine kinase, functions as a critical regulator of inflammatory pathways and mediates oncogenic events by phosphorylating its downstream targets extracellular signal regulated kinases (ERKs), c-Jun N-terminal kinases (JNKs) and P38 [9] and subsequently up-regulating the production of tumor necrosis factor- $\alpha$ (TNF- $\alpha$ ) and interleukin$1 \beta$ (IL-1 $\beta)$ [10]. The role of Tpl2 in acute and chronic inflammatory disorders and the determination of cellular death/survival ratios in the inflammatory microenvironment had been well-documented [11]. Our previous work demonstrated that whole-body ablation of Tpl2 attenuates high fat diet (HFD) induced hepatic inflammatory lesions compared to wild-type control mice, with a concomitant significant reduction in hepatic inflammatory genes expression [12]. However, the potential role of Tpl2 in tumorigenesis remains inconsistent $[11,13]$. Several reports supported its oncogenic role in breast cancer [14], lymphoma [15] and prostate cancer [16], while others had suggested an antioncogenic activity in lung [17], colitis-associated tumorigenesis [18] and skin tumorigenesis [19]. This inconsistency could be attributed to the complexity of Tpl2's role in terms of specific organs, different stages of carcinogenesis or the animal models used. However, there had no reports investigating the potential contribution of Tpl2 to HCC development by using Tpl2 knockout mouse model to date.

In the present study, we investigate the role of $T p l 2$ and its potential mechanisms in the development of hepatic steatosis, inflammation and tumors including HCC. Both wild-type and Tpl2 knockout male mice were initiated with [diethylnitrosamine (DEN)] at 2 weeks of age, and 4 weeks later, both groups mice were given the high carbohydrate diet (HCD) feeding for 24 weeks to induce hepatic steatosis, inflammation and HCC.

\section{Methods}

\section{Animals, diet, carcinogen and study design}

Tpl2 knockout mice were provided by Dr. Philip Tschilis (Tufts University) and backcrossed into C57BL/6J mice for $>10$ generations, as previously described [12, 20]. Genotype of animals was verified at weaning and again before the animals were killed at the conclusion of the experiment. Tpl2 mRNA expression was detected in wild-type but not in Tpl2 knockout mice (data not shown). All male C57BL/6J wild-type mice and Tpl2 knockout mice were injected i.p. with a single dose of 25 mg. $\mathrm{kg}^{-1}$ BW filter-sterilized, > $99.9 \%$ purity DEN (Sigma-Aldrich, St. Louis, MO) at 2 weeks after birth, as previously described [21]. At 6 weeks of age, both wildtype $(\mathrm{n}=26)$ and $T p l 2$ knockout $(\mathrm{n}=20)$ mice were housed individually and fed a powdered, high carbohydrate, low fat diet [HCD, $12 \%$ fat, $22 \%$ protein, $66 \%$ carbohydrates based on total caloric content (Bio-Serv, Flemington, NJ)] ad libitum for 24 weeks, as previously described [22]. Mice were killed by terminal exsanguination under deep isoflurane anesthesia followed by vital organ removal. All animal protocols and procedures were conducted under the approval of the Institutional Animal Care and Use Committee at the Jean Mayer USDA Human Nutrition Research Center on Aging at Tufts University. All animals received human care and that study protocols comply with the institution's guidelines.

\section{Histopathology procedures and evaluation}

Briefly, two investigators, blinded to the treatment groups, identified/counted the liver tumors (tumor incidence and multiplicity) on the surface of liver. The left lobe of the mouse liver was fixed in $10 \%$ buffered formalin solution (Thermo Fisher Scientific, Waltham $\mathrm{MA}$ ), processed and embedded in paraffin for serial sectioning for pathological analysis. The remaining lobes of the liver were divided into smaller portions, snap-frozen in liquid nitrogen and stored at $-80^{\circ} \mathrm{C}$.

Five-micrometer sections of liver tissue were stained with hematoxylin $(\mathrm{H})$ and eosin (E) (H\&E) for histopathologic examination. Two independent investigators, blinded to treatment groups, examined the sections under light microscopy and identified hepatic lesions (including hyperplasia, hepatocellular adenoma and HCC). Liver histopathology of non-tumor areas was graded for steatosis magnitude (both macro- and micro- vesicular) and liver inflammation severity (inflammatory foci) as previously described [21]. Briefly, the degree of steatosis was graded 0-3 [grading 0: $<5 \%$ (normal), 1: $5 \%-25 \%$, 2: $26 \%-50 \%, 3:>51 \%]$ based on the average percent of fat-accumulated hepatocytes per field at $100 \times$ magnification in 20 random fields. Inflammatory foci were evaluated by the number of inflammatory cell clusters (mononuclear inflammatory cells) in 20 random fields at $100 \times$ magnification. The twenty fields of view at $100 \times$ magnification represented $0.63 \mathrm{~cm}^{2}$ and inflammatory foci counts were represented as the number of foci per $\mathrm{cm}^{2}$. A ZEISS microscope with a PixeLINK USB 2.0 (PL-B623CU) digital Camera and PixeLINK $\mu$ Scope Microscopy Software (Ottawa ON, Canada) was used for image capture for all histological analyses.

\section{RNA extraction and real time-PCR}

RNA of liver tissue was isolated using TriPure Isolation Reagent (Roche, NJ) according to the manufacturer's instructions. cDNA was synthesized using M-MLV reverse 
transcriptase (Invitrogen, Grand Island, NY). Real-time PCR reactions were carried out using SYBR green (Fast Start Universal SYBR Green Master, Roche). Relative changes in gene expression were determined using the $2^{-\Delta \Delta \mathrm{Ct}}$ method and normalized to the control of actin.

\section{Western blot analysis}

Hepatic whole cell lysate protein was extracted utilizing previously described methods [23]. Protein concentrations of the sample were assessed using the Bradford assay (BioRad, Hercules CA). The protein expression bands were quantified using a densitometer (GS-710 calibrated imaging densitometer, Bio-Rad). The antibodies against acetyl-CoA carboxylase (ACC), stearoyl-CoA desaturase 1 (SCD-1), total- and phosphor- AKT (p-AKT, t-AKT), glucose regulated protein 78 (GRP78/Bip), total- and phosphor- (Thr980) PERK (t-PERK, p-PERK), total- and phosphor- (Ser51) eukaryotic translation initiation factor 2a (t-eIF2a, p-eIF2a), total- and phosphor- (Thr183/ Tyr185) JNK (p-JNK, t-JNK), total- and phosphor- ERK (p-ERK, t-ERK) were all purchased from Cell Signaling (Danvers, MA). The antibodies against sterol regulatory element-binding protein-1c (SREBP-1c) and C/EBP homologues protein (CHOP) were purchased from Santa Cruz Biotechnology (Dallas, TX).

\section{Statistical analyses}

Data are presented as mean \pm standard deviation (SD) for animal body weights, liver tumor numbers per animal and inflammatory foci, and nonparametric test was performed except for the comparison of the final body weights which was conducted using an independent $\mathrm{t}$-test. The incidence of hepatic lesions and inflammation foci was compared by Chi-squares test. Steatosis grading was presented as median (grading range) and a non-parametric test was performed. Data are presented as mean \pm standard error of the mean (SEM) for mRNA and protein levels, and t-test was conducted. SPSS software was used for all statistical analysis, and $P<0.05$ indicated the significant difference when comparing between wild-type and Tpl2 knockout mice.

\section{Results}

\section{Effect of $T p / 2$ ablation on body weight, liver weight and} hepatic tumorigenesis

Tpl2 knockout mice had significantly lower liver weight and final body weight than those of wild-type control, although there had no significant difference in the ratio of liver weight/body weight between two groups (Table 1). The tumor incidence on the surface of liver in Tpl2

Table 1 Study outcomes

\begin{tabular}{|c|c|c|c|c|}
\hline Index & Wild type group & & Tpl2 knockout group & \\
\hline Animal number (n) & 26 & & 20 & \\
\hline Final body weight (g) & $41.2 \pm 3.2$ & & $38.1 \pm 5.9^{*}$ & \\
\hline Liver weight (g) & $2.5 \pm 0.8$ & & $2.1 \pm 0.3^{*}$ & \\
\hline Liver/body weights (\%) & $6.2 \pm 2.4$ & & $5.6 \pm 1.1$ & \\
\hline Incidence of liver tumor (\%) & $100 \%(26 / 26)$ & & $75 \%(15 / 20)^{*}$ & \\
\hline Liver tumor number/per animal & $11 \pm 6$ & & $10 \pm 5$ & \\
\hline Histopathology of hepatic lesion" & $\mathrm{n}$ & $\%$ & $\mathrm{n}$ & $\%$ \\
\hline Hyperplasia & 26 & 100 & 3 & $15^{*}$ \\
\hline Hepatocellular adenoma & 26 & 100 & 12 & $60^{*}$ \\
\hline Hepatocellular carcinoma & 26 & 100 & 0 & $0^{*}$ \\
\hline Incidence of inflammation foci (\%) & $65.4 \%(17 / 26)$ & & $20 \%(4 / 20)^{*}$ & \\
\hline Inflammation foci number $\left(\mathrm{cm}^{2}\right)$ & $2.2 \pm 2.4$ & & $0.6 \pm 1.4^{*}$ & \\
\hline Hepatic steatosis (median) & 2 & & $1^{*}$ & \\
\hline Hepatic steatosis grading & $n$ & $\%$ & $n$ & $\%$ \\
\hline 0 & 1 & 3.8 & 8 & 40 \\
\hline 1 & 2 & 7.7 & 4 & 20 \\
\hline 2 & 15 & 57.7 & 4 & 20 \\
\hline 3 & 8 & 30.8 & 4 & 20 \\
\hline
\end{tabular}

${ }^{\text {"Each animal has more than one type of lesions }}$

Values are expressed as means \pm SD. An Independent t-test was performed except the comparison of incidence of liver tumor and inflammatory foci between WT and Tpl2 $\mathrm{KO}$ mice, which is conducted by nonparametric test. For steatosis, 20 images at $100 \times$ magnification were captured for each section and blindly evaluated twice to determine grade of steatosis (both macro- and micro-vesicular) by two blinded investigators. The degree of steatosis was graded 0-3 based on the area of the liver section occupied by fat vacuoles. Data are presented as median (grading range) and Nonparametric test was used to test for statistical significance between groups for ordinal variable (liver steatosis score)

For each given row, ${ }^{*}$ indicates a significant difference between groups $(P<0.05)$ 
knockout mice was significantly lower than wild-type mice (75\% vs $100 \%$ respectively, $P<0.05$, Table 1 ). The number of hepatic tumor had no statistical difference between those two groups. The pathological analysis demonstrated that all wild-type mice (26 out of total 26 mice) developed hyperplasia, hepatocellular adenoma (Fig. 1c, 1d) and HCC (Fig. 1e, 1f) after the 24-week HCD feeding (Table 1). In contrast, the Tpl2 knockout mice with positive tumor on the surface of the liver (15 out of 20) developed only hyperplasia and hepatocellular adenoma, and no HCC detected (Table 1).

Tp/2 ablation decreased hepatic inflammatory responses and suppressed the activation of JNK and ERK signaling molecules

Hepatic inflammatory foci was detected in $65 \%$ of the wild-type mice but only in $20 \%$ of the $T p l 2$ knockout mice $(P<0.05$; Table 1, Fig. 1 b). Tpl2 knockout mice had significantly less hepatic inflammatory foci in contrast to wild-type mice ( 0.6 vs $2.2, P<0.05$; Table 1 ), accompanying with lower mRNA level of genes $[I l-1 \beta$, interleukin18 (Il-18), monocyte chemotactic protein 1 (Mcp1), NACHT, LRR and PYD domains-containing protein 3 (Nalp3) related to hepatic inflammation (Fig. 2a)]. In addition, there was a significant decrease in the phosphorylation of JNK1/2 and ERK1/2, the downstream targets of TPL2-mediated inflammation signaling, in Tpl2 knockout mice as compared with wild-type mice (Fig. 2b).

Tp/2 ablation alleviated hepatic steatosis, and downregulated protein and mRNA expression of molecules involved in de novo lipogenesis (DNL) and endoplasmic reticulum (ER) stress

Tpl2 knockout mice had lower steatosis grades compared to wild-type mice (Table 1). Only one of 26 wild-

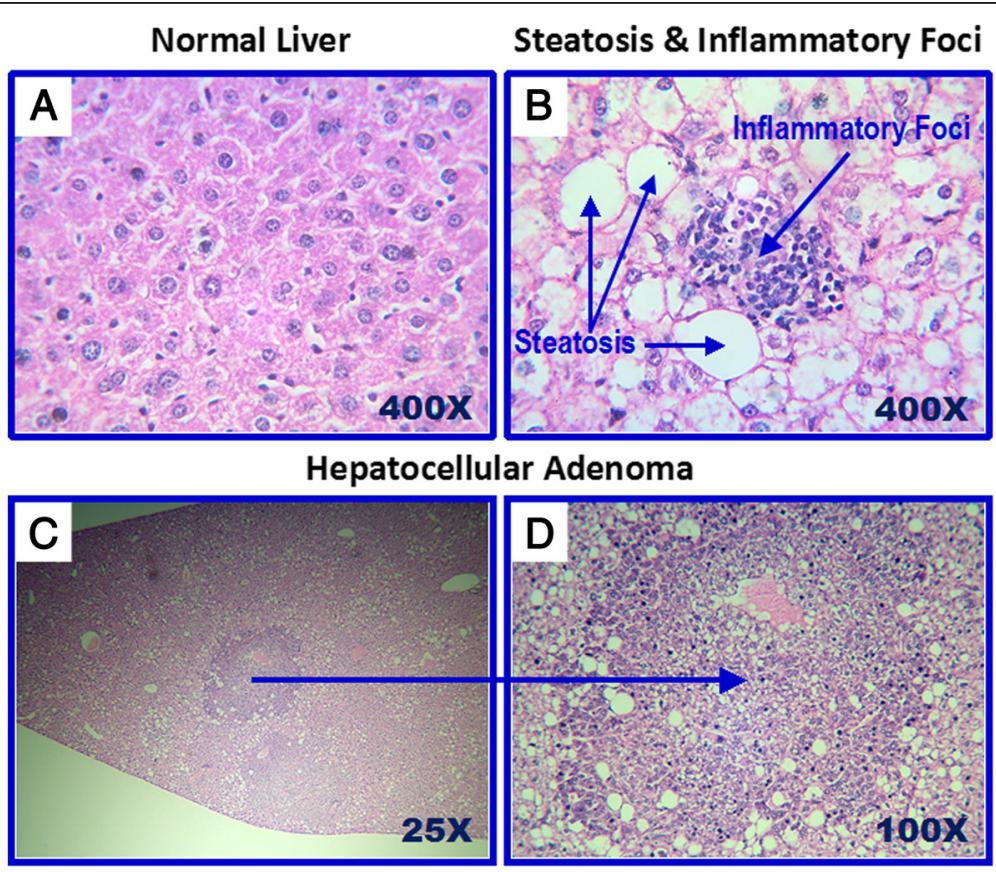

Hepatocellular Carcinoma

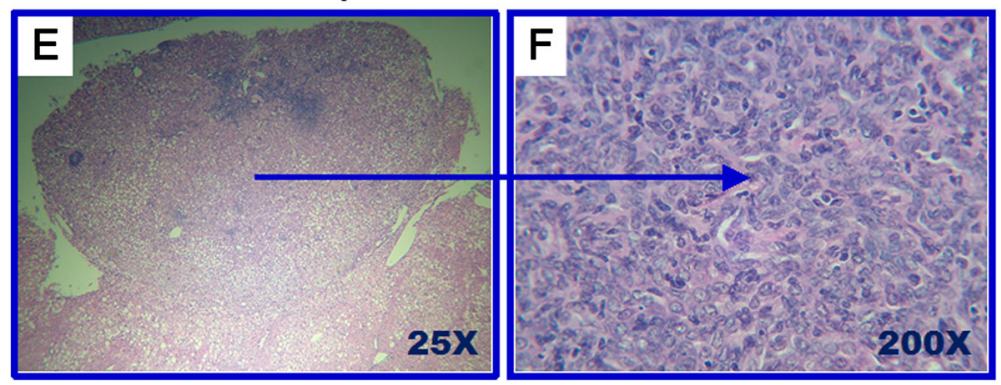

Fig. 1 Representative pathologic lesions in livers. Hepatic lesions were assessed by H\&E staining. Upper panel: Normal (Left); Steatosis and inflammatory foci (Right); Middle Panel: Hepatocellular adenoma (low magnification at x 25 and x100); Lower panel: HCC (low magnification at $\times 25$ and $\times 200$ ) 


\section{A}
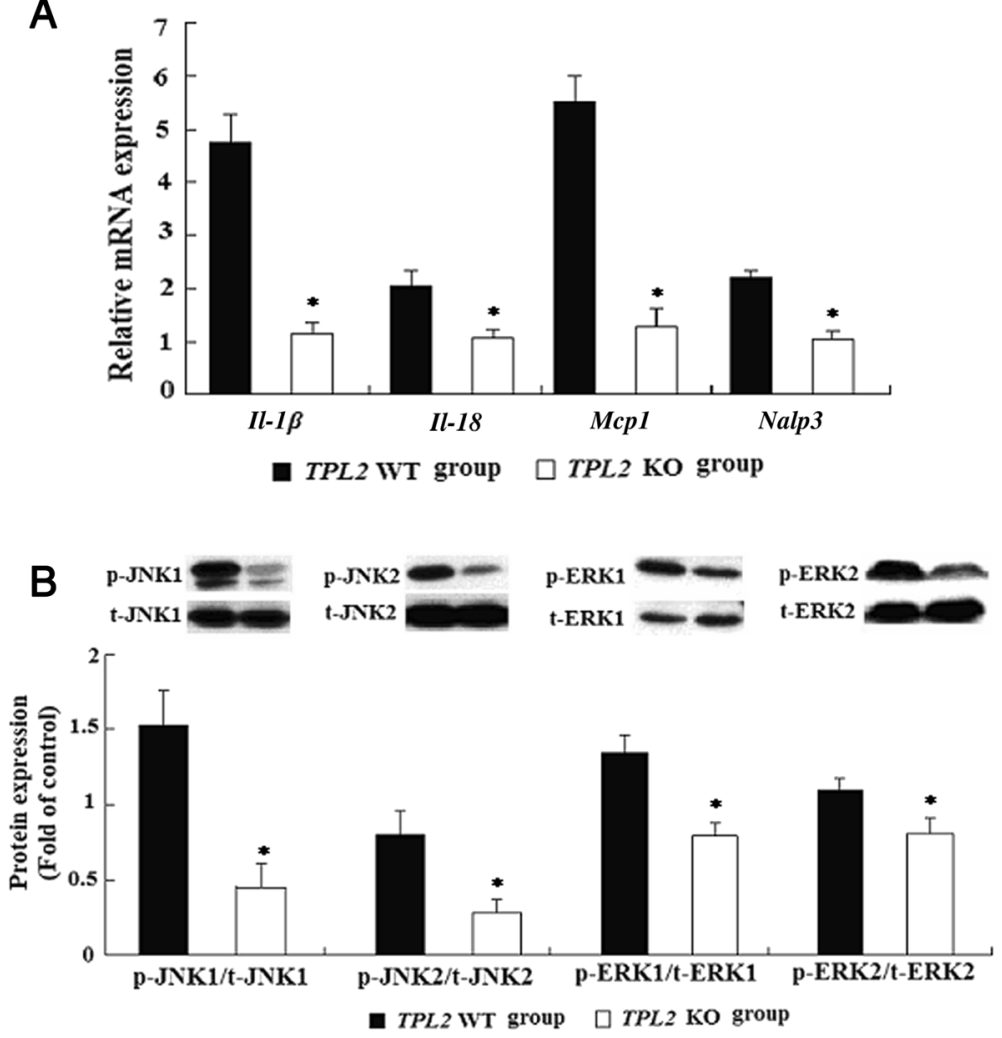

Fig. 2 Effect of Tp/2 ablation on hepatic mRNA expression of genes related to inflammation (a) and protein phosphorylations of JNK1/2 and ERK1/2 (b). a mRNA expression of genes related to inflammatory and macrophage markers in liver tissue in mice were detected by RT-PCR analysis. Values are expressed as mean \pm standard error of the mean (SEM). Actin was used as the control. $\mathbf{b}$ Proteins expression of JNK1/2 and ERK1/2 from liver tissue of Tp/2 knockout or wild-type mice were detected by western blotting analysis. Values are mean \pm standard error of the mean (SEM). *Comparing with Tp/2 wild type group. Insets: Representative pictures of western blotting analysis

type mice ( $3.8 \%)$ had a steatosis grading of 0 compared to 8 out of $20 \mathrm{Tpl} 2$ knockout mice (40\%). In contrast, 15 out of 26 wild-type mice (57.7\%) developed steatosis grade of 2 as compared to 4 out of $20 \mathrm{Tpl} 2$ knockout mice $(20 \%)$. Steatosis grades were statistically different between the 2 groups (medians of $2 v s$. 1 for wild-type vs. Tpl2 knockout, respectively, $P<0.05$, Table 1 ). In contrast to wild-type mice, Tpl2 knockout mice had significantly decreased proteins expression of ACC and SCD1, two lipogenic proteins (Fig. 3a), and decreased protein expression of SREBP1C (Fig. 3a), which is one of the transcription factors that regulates expression of genes involved in DNL. Meanwhile, we had detected the decreased expression of phosphorylated AKT (Fig. 3b), which is consistent with the decreased SREBP1c expression (Fig. 3a) and hepatic steatosis (Table 1) in Tpl2 knockout mice.

Increased ER stress can promote HCC development and progression by activating the fibrogenic activity of hepatic stellate cells with subsequent liver cirrhosis [24]. In the present study, Tpl2 knockout mice had decreased hepatic PERK and eIF2 $\alpha$ phosphorylation compared to wild-type mice (Fig. 4), but Tpl2 ablation did not alter the expression of the chaperon factor GRP78 that attenuates ER stress or PERK mediated pro-apoptotic protein CHOP (data not shown).

\section{Discussion}

The role of hepatic inflammation induced by dietary factors, such as HCD and HFD, in promoting DENinitiated HCC development had been demonstrated in previous studies $[6,22,25]$. The present study, for the first time, revealed the significantly lower incidence of hepatic tumor with no HCC development in HCD-fed, $T p l 2$ knockout mice in contrast to wild-type mice which all developed HCC. The significant difference in tumor pathological types between $T p l 2$ knockout mice and wild-type mice supported the critical role of $T p l 2$ as a promoter in tumor progression from hepatic hepatocellular adenoma to HCC. Furthermore, we provided a strong evidence that the TPL2 ablation decreased hepatic inflammatory response and hepatic steatosis in Tpl2 knockout mice. These effects could inhibit the malignant transformation of hepatocytes and the progression of 

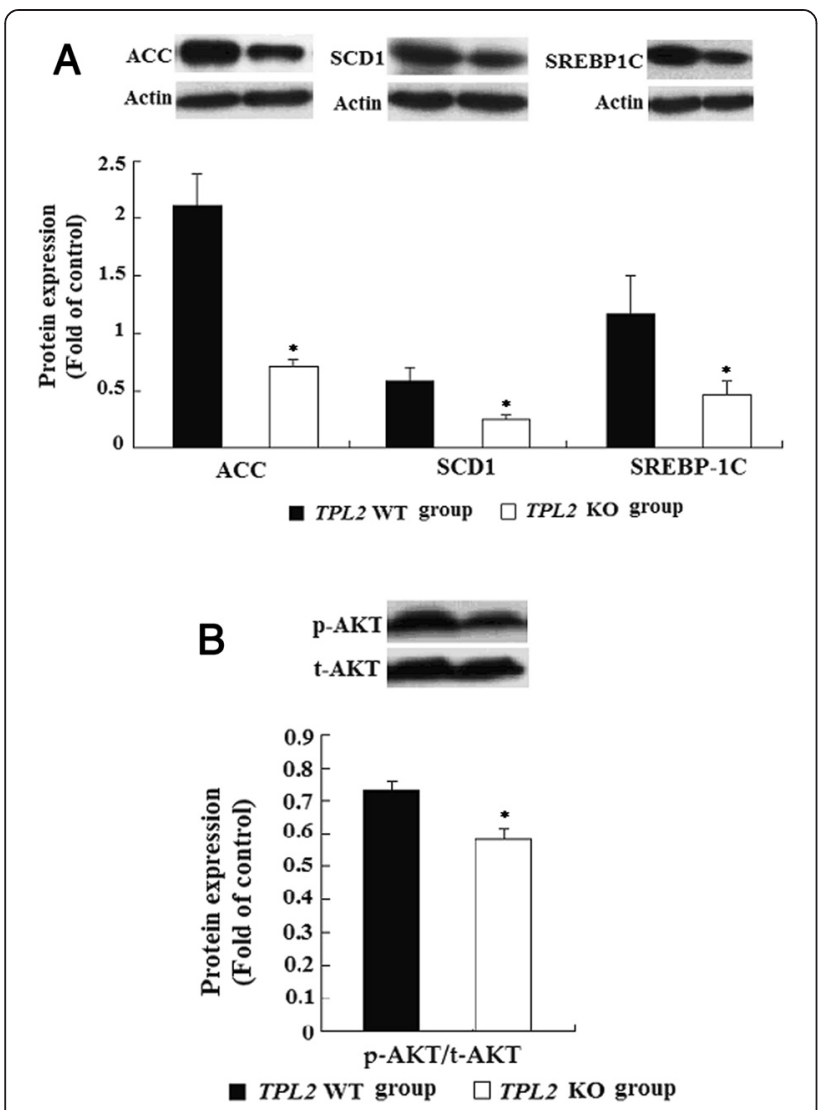

Fig. 3 Effect of Tp/2 ablation on hepatic protein expressions related to lipid metabolism (a) and AKT phosphorylation (b). Proteins expression related to lipogenesis (a) and AKT phosphorylation (b) from liver tissue were determined utilizing Western blotting analysis. Data are presented as mean \pm standard error of the mean (SEM). Actin was used as the control. *Comparing with Tp/2 wild type group. Insets: Representative pictures of western blotting analysis

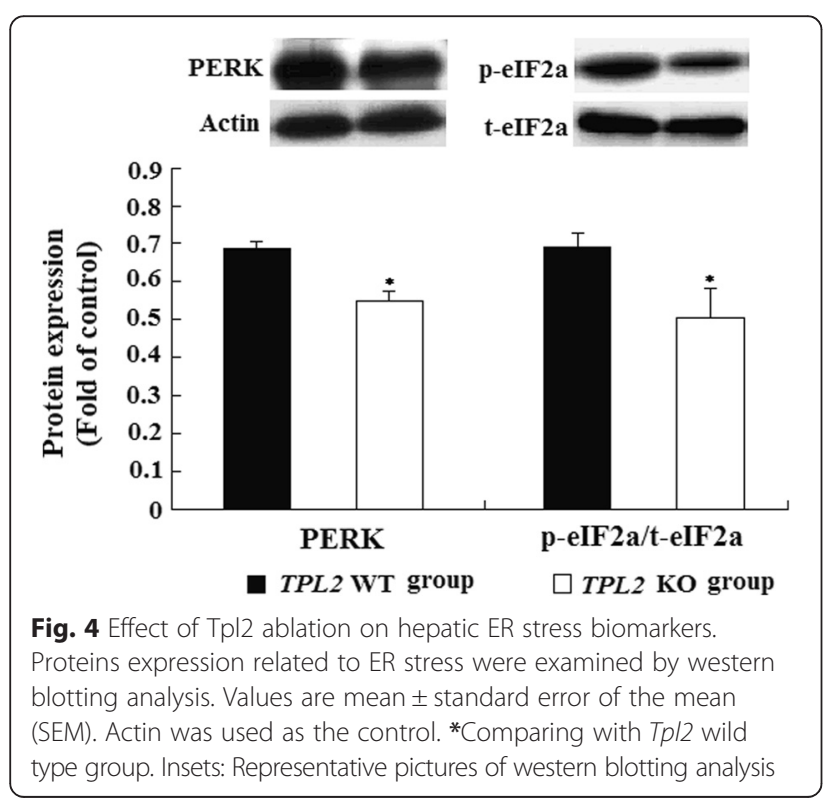

liver tumor by suppressing tumor-promoting microenvironment and alleviating the malignant effects of dys-regulation of lipid metabolism on HCC.

TPL2 mediated inflammatory response by phosphorylating ERK and JNK, two downstream targets of TPL2 signaling pathway. In our study, the significant lower protein levels of hepatic p-JNK and p-ERK in Tpl2 knock out mice was consistent with the decreased hepatic inflammation, which supported the role of activated ERK (p-ERK) and JNK (p-JNK) in mediating the pro-inflammatory effect of Tpl2. The decreased hepatic inflammation induced by HCD feeding supported by the fewer hepatic inflammatory foci detected and the lower levels of inflammatory cytokine expression of $\mathrm{Il}-1 \mathrm{~B}, \mathrm{Il}-18, \mathrm{Mcp}-1$ and Nalp3 in Tpl2 knockout mice, as compared with wide type mice. This is also in agreement with our previous study that $T p l 2$ knockout mice fed HFD had lower levels of inflammation compared to wild-type mice [12]. The present work further indicated the role of Tpl2 in mediating hepatic inflammation and HCC development induced by HCD.

Elevated hepatic DNL could promote hepatic steatosis [26]. Interestingly, in our present study, $T p l 2$ ablation resulted in a significant decrease of hepatic steatosis, and the down-regulated protein expression of genes related to DNL, such as ACC and SCD1, which was associated the decreased protein expression of AKT phosphorylation and SREBP-1C.

Previous studies have suggested that AKT activation is essential and sufficient to stimulate DNL and lipid accumulation through the induction of SREBP-1C [27, 28]. Furthermore, the promoting role of dysregulated lipid metabolism [29] and lipogenesis induced by activated AKT in HCC development had been documented [30]. Thus, the decrease in steatosis and AKT activation could further explain the decreased HCC in Tpl2 knockout mice.

However, the exact role of $T p l 2$ in regulating the genes related to DNL is unclear. It has been reported that the regulation of lipid metabolism and hepatic steatosis mediated by the activation of JNK, the downstream target of TPL2, is associated with activated ER stress, especially in the PERK-eIF2a pathway [31]. Our observation of the down-regulated expression of p-JNK, PERK and p-eIF2a in Tpl2 knockout mice with the decreased hepatic steatosis supported the involvement of ER stress in TPL2/ JNK mediated steatosis. Recent reports have demonstrated that ER stress is closely associated with hepatic lipogenesis with elevated DNL [26, 32, 33], and population-based studies also support the positive regulation of ER stress in hepatic lipogenesis [34, 35]. Since newly synthesized unfolded proteins in the ER is a major cause of activated ER stress and activated ER stress could further induce lipogenesis, the vicious cycle could result in the progression of steatosis [21]. In our present study, the deceased expression of the lipogenic enzymes 
ACC, SCD1 and SREBP-1C could decrease the impact of protein folding in the ER, and alleviate ER stress in Tpl2 knockout mice. PERK-mediated signaling can also promote apoptosis through inducing pro-apoptotic CHOP expression, the no significant difference of the protein level of $\mathrm{CHOP}$ suggested that HCD induced expression of PERK predominantly mediated lipogenesis, but not pro-apoptotic effects in our present study. Furthermore, the expression of GRP78, a chaperone protein that attenuates ER stress [36], were not different between the Tpl2 knockout and wild-type mice, combining with our observation that Tpl2 knockout mice had relatively lower levels of PERK and p-eIF2a, the downstream molecular of GRP78, than wild-type mice, we therefore concluded that $T p l 2$ mediated hepatic lipogenesis by targeting the axis of TPL2/JNK/ER stress/p-eIF2a, the downstream of GRP78.

Our previous study had shown that HCD feeding promoted DEN-initiated HCC development accompanying with the induction of the hepatic ER stressmediated PERK activation, which subsequently induced the elevated expression of pro-survival markers AKT and ERK1/2 [22]. Our present study demonstrated that Tpl2 ablation decreased ER stress mediated PERK expression and eIF-2a activation, which might account for the decreased tumor incidence. Since ER-dependent cell fate is associated with the activation of JNK/ERK [37], and the pro-survival role of activated ERK can be mediated by AKT phosphorylation and the involvement of PERK/eIF2a signaling [38-40], thus, in the present study, the down-regulated activation of ERK, AKT and PERK/eIF2a provided further explanation for the decreased incidence of HCC in Tpl2 knockout mice. The exact relationship between TPL2 and ER stress requires further investigation.

In summary, our present study demonstrated that $T p l 2$ played significant role in DEN-initiated, NAFLD associated HCC development by using Tpl2 knockout mouse model. Both TPL2/ERK/JNK axis mediated hepatic inflammation and TPL2/JNK/ERS/p-eIF2a axis mediated hepatic lipogenesis synergistically promoted HCC development. These data provided strong molecular evidence supporting Tpl2 as a promoter in HCC development. Interestingly, it has been shown that luteolin, one of the common phytonutrients present in celery, parsley, broccoli and herbal spices, could target Tpl2 and inhibit its activity in vitro [41]. Recent in vivo studies also supported the preventive effects of luteolin on DEN-initiated alcohol-promoted hepatic carcinogenesis in mice [42], and DEN-initiated HCC development in rats [43]. Taking all into consideration, dietary or pharmacologic interventions targeting Tpl 2 could be a potential direction for HCC prevention in the future.

\section{Conclusions}

This is the first study to report that tumor progression locus $2(\mathrm{Tpl} 2)$ plays a significant role in promoting nonalcoholic fatty liver disease and hepatocellular carcinoma (HCC) development. Targeting Tpl2 might be a potential direction for HCC prevention.

\section{Abbreviations}

HCC: hepatocellular carcinoma; NAFLD: non-alcoholic fatty liver disease; TG: triglycerides; NASH: nonalcoholic steatohepatitis; TPL2: tumor progression locus 2; MAP3K8: MAP3 kinase 8; ERKs: extracellular signal regulated kinases; JNKs: c-jun N-terminal kinases; TNF-a: tumor necrosis factor-a; IL-1 $\beta$ : interleukin-1 $\beta$; HFD: high fat diet; DEN: diethylnitrosamine; HCD: high carbohydrates diet; AKT: protein kinase B; ER: endoplasmicreticulum; PERK: PKR-like kinase; ACC: acetyl-CoA carboxylase; SCD: stearoyl-CoA desaturase; p-: phosphorylated; t-: total; GRP78: Glucose regulated protein 78; elF2a: eukaryotic translation initiation factor 2a; SREBP-1c: sterol regulatory element-binding protein-1c; CHOP: C/EBP homologues protein; SD: standard deviation; SEM: standard error of the mean; IL-18: Interleukin-18; MCP-1: monocyte chemotactic protein 1; NALP3: NACHT, LRR and PYD domains-containing protein 3; DNL: de novo lipogenesis.

\section{Competing interests}

The authors declare no conflicts of interest.

\section{Authors' contributions}

XLL and KQH conducted RT-PCR and Western blot. CL participated in the histo-pathological analysis. DES and ASG participated in the animal breeding. $\mathrm{BCl}$ and XDW participated in the design of the study. XLL performed the statistical analysis. XLL and XDW drafted the manuscript. All authors read and approved the final manuscript.

\section{Acknowledgments}

The author thanks Dr. Camilla P. Stice, and the HNRCA Cancer Cluster members for their contributions to this study. Dr. Xinli Li was supported by a scholarship (File NO. 2011832272) from the State Scholarship Fund by The China Scholarship Council.

Financial support was provided by the NIH grant (CA176256), USDA/ARS grant (58-1950-0014), and HNRCA Cancer Cluster funding.

\section{Author details}

${ }^{1}$ Nutrition and Cancer Biology Laboratory, Jean Mayer USDA Human Nutrition Research Center on Aging at Tufts University, 711 Washington Street, Boston, MA 02111, USA. ${ }^{2}$ Comparative Biology Unit, Boston, MA, USA. ${ }^{3}$ Obesity and Metabolism Laboratory, Jean Mayer USDA Human Nutrition Research Center on Aging at Tufts University, Boston, MA 02111, USA. ${ }^{4}$ School of Public Health, Medical College of Soochow University, Suzhou, Jiangsu 215123, PR China.

Received: 16 September 2015 Accepted: 4 November 2015 Published online: 11 November 2015

\section{References}

1. Jemal A, Bray F, Center MM, Ferlay J, Ward E, Forman D. Global cancer statistics. CA Cancer J Clin. 2011;61(2):69-90. doi:10.3322/caac.20107.

2. El-Serag HB, Rudolph KL. Hepatocellular carcinoma: epidemiology and molecular carcinogenesis. Gastroenterology. 2007;132(7):2557-76. doi:10.1053/j.gastro.2007.04.061.

3. Mittal S, Sada YH, El-Serag HB, Kanwal F, Duan Z, Temple S, et al. Temporal Trends of Nonalcoholic Fatty Liver Disease-Related Hepatocellular Carcinoma in the Veteran Affairs Population. Clin Gastroenterol Hepatol. 2014. doi:10.1016/j.cgh.2014.08.013.

4. Baffy G, Brunt EM, Caldwell SH. Hepatocellular carcinoma in non-alcoholic fatty liver disease: an emerging menace. J Hepatol. 2012;56(6):1384-91. doi:10.1016/j.jhep.2011.10.027.

5. Duan XY, Zhang L, Fan JG, Qiao L. NAFLD leads to liver cancer: do we have sufficient evidence? Cancer Lett. 2014;345(2):230-4. doi:10.1016/ j.canlet.2013.07.033.

6. Hernandez-Gea V, Toffanin S, Friedman SL, Llovet JM. Role of the microenvironment in the pathogenesis and treatment of hepatocellular 
carcinoma. Gastroenterology. 2013;144(3):512-27. doi:10.1053/ j.gastro.2013.01.002.

7. Sun B, Karin M. Obesity, inflammation, and liver cancer. J Hepatol, 2012;56(3):704-13. doi:10.1016/j.jhep.2011.09.020.

8. Adams LA, Lymp JF, St Sauver J, Sanderson SO, Lindor KD, Feldstein A, et al. The natural history of nonalcoholic fatty liver disease: a population-based cohort study. Gastroenterology. 2005;129(1):113-21.

9. Aoki M, Akiyama T, Miyoshi J, Toyoshima K. Identification and characterization of protein products of the cot oncogene with serine kinase activity. Oncogene. 1991;6(9):1515-9.

10. Yaomura T, Tsuboi N, Urahama Y, Hobo A, Sugimoto K, Miyoshi J, et al. Serine/threonine kinase, $\mathrm{Cot} / \mathrm{Tp} \mathrm{L}$, regulates renal cell apoptosis in ischaemia/reperfusion injury. Nephrology (Carlton). 2008;13(5):397-404. doi:10.1111/j.1440-1797.2008.00959.x.

11. Vougioukalaki M, Kanellis DC, Gkouskou K, Eliopoulos AG. Tpl2 kinase signal transduction in inflammation and cancer. Cancer Lett. 2011;304(2):80-9. doi:10.1016/j.canlet.2011.02.004

12. Perfield 2nd JW, Lee Y, Shulman GI, Samuel VT, Jurczak MJ, Chang E, et al. Tumor progression locus 2 (TPL2) regulates obesity-associated inflammation and insulin resistance. Diabetes. 2011;60(4):1 168-76. doi:10.2337/db10-0715.

13. Lee HW, Choi HY, Joo KM, Nam DH. Tumor progression locus 2 (Tpl2) kinase as a novel therapeutic target for cancer: double-sided effects of Tpl2 on cancer. Int J Mol Sci. 2015;16(3):4471-91. doi:10.3390/ijms16034471.

14. Kim G, Khanal P, Kim JY, Yun HJ, Lim SC, Shim JH, et al. COT phosphorylates prolyl-isomerase Pin1 to promote tumorigenesis in breast cancer. Mol Carcinog. 2013. doi:10.1002/mc.22112.

15. Christoforidou AV, Papadaki HA, Margioris AN, Eliopoulos GD, Tsatsanis C. Expression of the Tpl2/Cot oncogene in human T-cell neoplasias. Mol Cancer. 2004;3(1):34. doi:10.1186/1476-4598-3-34.

16. Lee HW, Cho HJ, Lee SJ, Song HJ, Park MC, Seol HJ, et al. Tpl2 induces castration resistant prostate cancer progression and metastasis. Int J Cancer. 2014. doi:10.1002/ijc.29248.

17. Gkirtzimanaki K, Gkouskou KK, Oleksiewicz U, Nikolaidis G, Vyrla D, Liontos $M$, et al. TPL2 kinase is a suppressor of lung carcinogenesis. Proc Natl Acad Sci U S A. 2013;110(16):E1470-9. doi:10.1073/pnas.1215938110.

18. Koliaraki V, Roulis M, Kollias G. Tpl2 regulates intestinal myofibroblast HGF release to suppress colitis-associated tumorigenesis. J Clin Invest. 2012;122(11):4231-42. doi:10.1172/jci63917.

19. DeCicco-Skinner KL, Nolan SJ, Deshpande MM, Trovato EL, Dempsey TA, Wiest JS. Altered prostanoid signaling contributes to increased skin tumorigenesis in Tpl2 knockout mice. PLoS One. 2013;8(2), e56212. doi:10.1371/journal.pone.0056212.

20. Dumitru CD, Ceci JD, Tsatsanis C, Kontoyiannis D, Stamatakis K, Lin JH, et al. TNF-alpha induction by LPS is regulated posttranscriptionally via a Tpl2/ERKdependent pathway. Cell. 2000;103(7):1071-83.

21. Ip BC, Hu KQ, Liu C, Smith DE, Obin MS, Ausman LM, et al. Lycopene metabolite, apo-10'-lycopenoic acid, inhibits diethylnitrosamine-initiated, high fat diet-promoted hepatic inflammation and tumorigenesis in mice. Cancer Prev Res (Phila). 2013;6(12):1304-16. doi:10.1158/1940-6207.capr-13-0178.

22. Ip BC, Liu C, Smith DE, Ausman LM, Wang XD. High-refined-carbohydrate and high-fat diets induce comparable hepatic tumorigenesis in male mice. J Nutr. 2014;144(5):647-53. doi:10.3945/jn.113.189613.

23. Xie $B$, Lin $W$, Ye J, Wang $X$, Zhang B, Xiong $S$, et al. DDR2 facilitates hepatocellular carcinoma invasion and metastasis via activating ERK signaling and stabilizing SNAIL1. J Exp Clin Cancer Res. 2015;34(1):101. do::10.1186/s13046-015-0218-6.

24. Hernandez-Gea V, Hilscher M, Rozenfeld R, Lim MP, Nieto N, Werner S, et al. Endoplasmic reticulum stress induces fibrogenic activity in hepatic stellate cells through autophagy. J Hepatol. 2013;59(1):98-104. doi:10.1016/ j.jhep.2013.02.016.

25. Rius B, Lopez-Vicario C, Gonzalez-Periz A, Moran-Salvador E, Garcia-Alonso V, Claria J, et al. Resolution of inflammation in obesity-induced liver disease. Front Immunol. 2012;3:257. doi:10.3389/fimmu.2012.00257.

26. Ren LP, Chan SM, Zeng XY, Laybutt DR, Iseli TJ, Sun RQ, et al. Differing endoplasmic reticulum stress response to excess lipogenesis versus lipid oversupply in relation to hepatic steatosis and insulin resistance. PLoS One. 2012;7(2), e30816. doi:10.1371/journal.pone.0030816.

27. Krycer JR, Sharpe LJ, Luu W, Brown AJ. The Akt-SREBP nexus: cell signaling meets lipid metabolism. Trends Endocrinol Metab. 2010;21(5):268-76. doi:10.1016/j.tem.2010.01.001.
28. Fleischmann M, lynedjian PB. Regulation of sterol regulatory-element binding protein 1 gene expression in liver: role of insulin and protein kinase B/cAkt. Biochem J. 2000;349(Pt 1):13-7.

29. Park EJ, Lee JH, Yu GY, He G, Ali SR, Holzer RG, et al. Dietary and genetic obesity promote liver inflammation and tumorigenesis by enhancing IL-6 and TNF expression. Cell. 2010;140(2):197-208. doi:10.1016/j.cell.2009.12.052.

30. Nakanishi K, Sakamoto M, Yamasaki S, Todo S, Hirohashi S. Akt phosphorylation is a risk factor for early disease recurrence and poor prognosis in hepatocellular carcinoma. Cancer. 2005;103(2):307-12. doi:10.1002/cncr.20774.

31. Lee AH, Scapa EF, Cohen DE, Glimcher LH. Regulation of hepatic lipogenesis by the transcription factor XBP1. Science. 2008;320(5882):1492-6. doi:10.1126/science.1158042.

32. Lee AH, Glimcher LH. Intersection of the unfolded protein response and hepatic lipid metabolism. Cell Mol Life Sci. 2009;66(17):2835-50. doi:10.1007/ s00018-009-0049-8.

33. Glimcher $L H$, Lee $A H$. From sugar to fat: How the transcription factor XBP1 regulates hepatic lipogenesis. Ann N Y Acad Sci. 2009;1173 Suppl 1:E2-9. doi:10.1111/j.1749-6632.2009.04956.X.

34. Boden G, Duan X, Homko C, Molina EJ, Song W, Perez O, et al. Increase in endoplasmic reticulum stress-related proteins and genes in adipose tissue of obese, insulin-resistant individuals. Diabetes. 2008;57(9):2438-44. doi:10.2337/db08-0604.

35. Das SK, Chu WS, Mondal AK, Sharma NK, Kern PA, Rasouli N, et al. Effect of pioglitazone treatment on endoplasmic reticulum stress response in human adipose and in palmitate-induced stress in human liver and adipose cell lines. Am J Physiol Endocrinol Metab. 2008;295(2):E393-400. doi:10.1152/ ajpendo.90355.2008.

36. Ni M, Lee AS. ER chaperones in mammalian development and human diseases. FEBS Lett. 2007;581(19):3641-51. doi:10.1016/j.febslet.2007.04.045.

37. Oh SH, Lim SC. Endoplasmic reticulum stress-mediated autophagy/ apoptosis induced by capsaicin (8-methyl-N-vanillyl-6-nonenamide) and dihydrocapsaicin is regulated by the extent of c-Jun NH2-terminal kinase/ extracellular signal-regulated kinase activation in WI38 lung epithelial fibroblast cells. J Pharmacol Exp Ther. 2009;329(1):112-22. doi:10.1124/ jpet.108.144113.

38. Calvisi DF, Wang C, Ho C, Ladu S, Lee SA, Mattu S, et al. Increased lipogenesis, induced by AKT-mTORC1-RPS6 signaling, promotes development of human hepatocellular carcinoma. Gastroenterology. 2011;140(3):1071-83. doi:10.1053/j.gastro.2010.12.006.

39. Koumenis C, Naczki C, Koritzinsky M, Rastani S, Diehl A, Sonenberg N, et al. Regulation of protein synthesis by hypoxia via activation of the endoplasmic reticulum kinase PERK and phosphorylation of the translation initiation factor elF2alpha. Mol Cell Biol. 2002;22(21):7405-16.

40. Mounir Z, Krishnamoorthy JL, Wang S, Papadopoulou B, Campbell S, Muller WJ et al. Akt determines cell fate through inhibition of the PERK-elF2alpha phosphorylation pathway. Sci Signal. 2011;4(192):ra62. doi:10.1126/ scisignal.2001630.

41. Kim JE, Son JE, Jang YJ, Lee DE, Kang NJ, Jung SK, et al. Luteolin, a novel natural inhibitor of tumor progression locus 2 serine/threonine kinase, inhibits tumor necrosis factor-alpha-induced cyclooxygenase-2 expression in JB6 mouse epidermis cells. J Pharmacol Exp Ther. 2011;338(3):1013-22. doi:10.1124/jpet.111.179200.

42. Rafacho CPS BPM, Liu C, Greenberg AS, Ausman LM, Wang XD. Inhibition of diethylnitrosamine-initiated alcohol-promoted hepatic inflammation and precancerous lesions by flavonoid luteolin is associated with increased sirtuin 1 activity in mice. HepatoBiliary Sur Nutr. 2015;4:124-34.

43. Balamurugan $\mathrm{K}$, Karthikeyan J. Evaluation of Luteolin in the Prevention of $\mathrm{N}$-nitrosodiethylamine-induced Hepatocellular Carcinoma Using Animal Model System. Indian J Clin Biochem. 2012;27(2):157-63. doi:10.1007/ s12291-011-0166-7. 